\title{
DEVELOPMENTAL CHANGES IN MESENTERIC ARTERY REACTIVITY IN FETAL AND
} PERINATAL CHICKS

\author{
R.M. Moonen ${ }^{1}$, L. Brinks ${ }^{2}$, E. Villamor ${ }^{2}$ \\ ${ }^{I}$ Pediatrics, Atrium Medical Center, Heerlen, ${ }^{2}$ Pediatrics, Maastricht University Medical Center (MUMC+), \\ GROW School, Maastricht, The Netherlands
}

Background and aims: The fetal intestine presents a low-flow, high-resistance vascular bed. Birth is accompanied by a dramatic increase in intestinal blood flow. The vascular changes accompanying this transition have been partly characterized. Here, we aimed to investigate the developmental changes in chick mesenteric artery (MA) reactivity during the fetal and perinatal period.

Methods: Rings of the distal segment of the cranial MA from 15-d (F15) and 19-d (F19) chicken fetuses (total incubation: 21d) as well as non-fed 0-3-h-old (NH3h) and first-fed 1-d-old (NH1d) newly hatched $(\mathrm{NH})$ chicks were mounted in a wire myograph.

Results: F15 MA rings responded to $\mathrm{KCl}$-evoked depolarization and to norepinephrine (NE), thromboxane $\mathrm{A}_{2}$ (U46619), endothelin (ET)-1, acetylcholine (ACh), sodium nitroprusside (SNP), and forskolin indicating the presence of electro- and pharmaco-mechanical coupling as well as cGMP- and cAMP-mediated relaxation. The contractile efficacy of $\mathrm{KCl}$ underwent a linear increase $(\mathrm{F} 15<\mathrm{F} 19<\mathrm{NH} 3 \mathrm{~h}<\mathrm{NH} 1 \mathrm{~d})$. The efficacy of NE and U46619 increased in ovo but not ex ovo $(\mathrm{F} 15<\mathrm{F} 19=\mathrm{NH} 3 \mathrm{~h}=\mathrm{NH} 1 \mathrm{~d})$ and the efficacy of ET-1 peaked at F19. The relaxations elicited by ACh, SNP, and forskolin did not undergo dramatic developmental changes. Hypoxia induced contraction in MA rings. F15 MAs did not respond to hypoxia and the hypoxic responsiveness did not change ex-ovo $(\mathrm{F} 15=0<\mathrm{F} 19<\mathrm{NH} 3 \mathrm{~h}=\mathrm{NH} 1 \mathrm{~d})$.

Conclusions: The ability of chick MAs to constrict in response to pharmacological and physical stimuli increases during the fetal period but no dramatic changes are induced by hatching or the first feeding. Maturation of vasodilator mechanisms precedes that of vasoconstrictor mechanisms. 